\title{
Estudio preliminar de líquenes del orden Peltigerales presentes en el Parque Ecológico y Cultural Rucamanque, Región de La Araucanía, Chile
}

\author{
Preliminary study of lichens of the order Peltigerales in the Ecological and Cultural Park \\ Rucamanque in the Araucania Region, Chile
}

\section{Johana Villagra ${ }^{1}$, Manuel Muñoz-Muñoz ${ }^{1}$, Patricio Nuñez ${ }^{3}$ \& Angélica Casanova-Katny,1,2,*}

'Laboratorio de Ecofisiología Vegetal y Cambio Climático, Facultad de Recursos Naturales, Universidad Católica de Temuco, Temuco, Chile.

${ }^{2}$ Núcleo de estudios ambientales (NEA), Facultad de Recursos Naturales, Universidad Católica de Temuco, Temuco, Chile.

${ }^{3}$ Departamento de Cs. Forestales, Universidad de La Frontera, Temuco, Chile.

*E-mail: mcasanova@uct.cl

\begin{abstract}
The Rucamanque Park houses some of the remnants of the Roble-Raulí-Coigüe mixed forest in CentralSouth Chile. Cortical lichens were monitored in Renoval and in the Original Remnant Forest of Nothofagus obliqua (Mirb.) Oerst. We found differences in specific richness by tree species and between the Renoval (13 species) and the Original Remnant (7 species). The variables that affect the greatest richness are discussed, suggesting that the structure of the forest may play a key factor.
\end{abstract}

\section{INTRODUCCIÓN}

Los líquenes son organismos complejos producto de la asociación simbiótica, entre un hongo (micobionte) y un organismo fotosintético (fotobionte), que bien puede ser, un alga verde o una cianobacteria, o ambos (Nash 2008). Los líquenes corticícolas son diversos y funcionalmente importantes en los ecosistemas forestales, donde contribuyen a la biomasa y juegan un papel clave en el ciclo del agua y los nutrientes (Van Stan \& Pypker 2015; Asplund \& Wardle 2016). La diversidad de líquenes corticícolas está relacionada con el tipo de bosque, la edad, la composición y la estructura, así como con la disponibilidad de luz y humedad entre otros factores (Lesica et al. 1991; Armstrong \& Welch 2007; Fanning et al. 2007; Nascimbene et al. 2009; Moning et al. 2009; Johansson et al. 2010; Pinho et al. 2012; Li et al. 2015; Cardós et al. 2016). La distribución de líquenes en los árboles no es homogénea, viene determinada por el microhábitat, y se ha observado que algunas especies muestran preferencia por zonas sombreadas y húmedas, mientras que otras por zonas de mayor exposición, además el rango de tolerancia de estas variables microclimáticas es variable entre especies (Sporn et al. 2010). Así también la altura de los árboles genera cambios en la diversidad liquénica, por las diferencias en variables microambientales o la contaminación atmosférica, por sobre todo el aumento en nitrógeno (Prather et al. 2018). La mayoría de las especies del orden Peltigerales presentan cianobacterias como fotobionte en sus talos, tanto en especies bipartitas (un micobionte, un fotobionte) como en especies tripartitas (un micobionte, dos fotobiontes) donde están restringidos a cefalodios y un alga verde es el fotobionte principal (Miadlikowska \& Lutzoni 2004). Aunque el orden Peltigerales cuenta con 67 géneros y más de 1300 especies en el mundo (Lücking et al. 2017a), de acuerdo a la Lista de Líquenes de Chile recientemente actualizada, este orden está representado en nuestro país por 9 familias y al menos 42 géneros (Vargas \& Sandoval 2020), siendo la familia más numerosa la Pannariaceae (20), Lobariaceae (8), 
Crococarpiaceae (3) Peltigeraceae (3), Placynthiaceae (3), Collemataceae (2) y las restantes familias representadas por un género (Nephromatacea, Massolongiaceae, Koerberiaceae). Algunos estudios en el sur de Chile muestran un alto número de líquenes corticícolas en diversas áreas protegidas privadas o estatales (Redón et al. 1979; Pereira 2007; Quilhot et al. 2010; Quilhot et al. 2012; Rubio et al. 2013; Pereira et al. 2016). Sin embargo, se desconocen estudios de criptógamas en la depresión intermedia del Centro-Sur de Chile, por lo que existe interés de efectuar estudios liquenológicos en este frágil ecosistema que ha sido fuertemente intervenido desde los tiempos de la colonización (Donoso 1983) y que actualmente están considerados como formaciones vegetacionales con riesgo crítico (Fernández et al. 2010). Así el objetivo de este trabajo fue elaborar un catálogo preliminar de líquenes epífitos del Orden Peltigerales presentes en un Remanente de Bosque Original y en un Renoval de Roble de la depresión intermedia del centro-sur de Chile y mostrar algunos patrones generales de la distribución de estos organismos.

\section{MATERIALES Y MÉTODOS}

\section{DEsCRIPCIÓN ÁREA DE ESTUDIO}

El estudio se llevó a cabo en el Parque Ecológico y Cultural Rucamanque (Fig. 1; 38³9'34" S 72³6'20,2" O) que es un predio perteneciente a Bienes Nacionales administrado por la Universidad de La Frontera (UFRO), emplazado en una quebrada con orientación Sureste a Noroeste del cordón montañoso Huimpil-Ñielol, con una altitud que fluctúa entre los 200 m s.n.m y 550 m s.n.m (Nuñez et al. 2015). El parque posee una superficie de $4.081 .310 \mathrm{~m}^{2}$, de los cuales aproximadamente $2.660 .000 \mathrm{~m}^{2}$ corresponden a bosques primarios o remanentes originales, compuestos principalmente por las especies arbóreas Nothofagus obliqua (Mirb.) Oerst., Nothofagus dombeyi (Mirb.) Oerst., seguido en algunos casos de Aextoxicon punctatum Ruiz \& Pav., Laurelia sempervirens (Ruiz \& Pav.) Tul. y Persea lingue (Ruiz \& Pav.) Nees como especies dominantes y codominantes (Donoso 1981) y $840.000 \mathrm{~m}^{2}$ de bosques secundarios de la misma composición (Nuñez et al. 2015). El Parque concentra el área mejor conservada y la mayor superficie de los bosques originales que existían en la depresión intermedia de la Región de la Araucanía de Chile. Se localiza a $12 \mathrm{~km}$ al noroeste de la ciudad de Temuco en la provincia de Cautín, Región de la Araucanía (Salas 2001). El clima del área es del tipo templado húmedo (Di Castri \& Hajek 1976), con una precipitación promedio anual que alcanza los $1.400 \mathrm{~mm}$ y una temperatura de $12{ }^{\circ} \mathrm{C}$, respectivamente. Las Iluvias se concentran en invierno, dejando 1 o 2 meses de sequía estival (Hajek \& Di Castri 1975).

\section{MÉTOdo de MUESTREO}

El muestreo se realizó en el sector oeste del Parque Rucamanque, en el mes de octubre del 2018 (Fig. 1). Se trabajó en dos tipos de bosque: un Bosque Remanente Original y un bosque renoval de Roble (Nothofagus obliqua). Se censaron individuos vivos de cuatro especies arbóreas dominantes, entre ellas A. punctatum, $P$. lingue, $N$. obliqua y $N$. dombeyi de DAP superiores a $40 \mathrm{~cm}$, por su cara en exposición norte, desde los 0 a $150 \mathrm{~cm}$ de altura en cuadrantes de 625 $\mathrm{cm}^{2}(25 \times 25 \mathrm{~cm})$. La recolección del material se centró en líquenes foliosos y gelatinosos (de acuerdo a Barreno \& PérezOrtega 2003) del Orden Peltigerales. Se censaron todos los forófitos presentes en un área total de $314 \mathrm{~m}^{2}$ (cada parcela circular de $10 \mathrm{~m}$ de radio aproximadamente). En el bosque Remanente Original fueron seleccionados cuatro especies arbóreas: Nothofagus obliqua, Nothofagus dombeyi, Persea lingue y Aextoxicon punctatum. En el renoval de Roble se seleccionaron las especies Nothofagus obliqua, Persea lingue y Aextoxicon punctatum. El número de árboles necesarios para obtener la máxima representación de especies de líquenes fue obtenido a través de una curva área/especie (Colwell et al. 2004), considerándose entre 5 y 15 individuos por especie de árbol, dependiendo de la accesibilidad, y centrándose sólo en Peltigerales (datos no mostrados).

Para los análisis de diversidad se utilizó el programa PAST 3 (versión 3.16), comparándose los registros entre dos sectores: Bosque Remanente Original y Bosque Renoval de Roble, obteniéndose la riqueza de cada sector: $(\mathrm{S}=$ número de especies diferentes en cada sector); Registros totales ( $n=$ Total de registros obtenidos en cada sector), Dominancia (donde ni es el número de individuos del taxón i), si $D=0$ representa que todos los taxones están representados de igual manera y $D=1$ representa que un taxón domina por completo la comunidad; Índice de diversidad de Simpson (Simpson = 1-D, midiendo la igualdad de comunidad de 0 a 1); y el Índice de diversidad de Shannon (índice que varía desde 0 para comunidades con solo un taxón hasta valores más altos para comunidades con muchos taxones, cada uno con pocos individuos).

\section{IDENTIFICACIÓN DE ESPECIES}

Para la identificación de las especies se utilizaron claves dicotómicas de Galloway $(1989,1992,1994)$ y White \& James (1988). Se utilizó una lupa estereoscópica (Zeiss, Stemi 2000) para la observación y medición de caracteres morfológicos externos y microscopio óptico (Olympus CX31, con magnificación de 1000x) para la observación de cortes al apotecio y secciones de talo. La identificación de los líquenes también incluyó test químicos (spot test) que determinan 
la presencia o ausencia de determinadas substancias en distintas estructuras del talo liquénico (Orange et al. 2001). Para ello se utilizó los reactivos K (solución saturada de $\mathrm{KOH}$ al 15\%), C (solución acuosa de hipoclorito de sodio al 5\%) y KC (aplicación de K seguida de C). Para la nomenclatura taxonómica se siguió Lücking et al. (2016), además de cotejar actualizaciones en Index fungorum. La distribución geográfica de las especies se determinó de acuerdo a Galloway (2008). Los especímenes recolectados han sido secados al aire por un día y guardados en bolsas de papel. Los especímenes identificados fueron depositados en el fungario CONC-F de la Universidad de Concepción, a cargo del Dr. Götz Palfner.
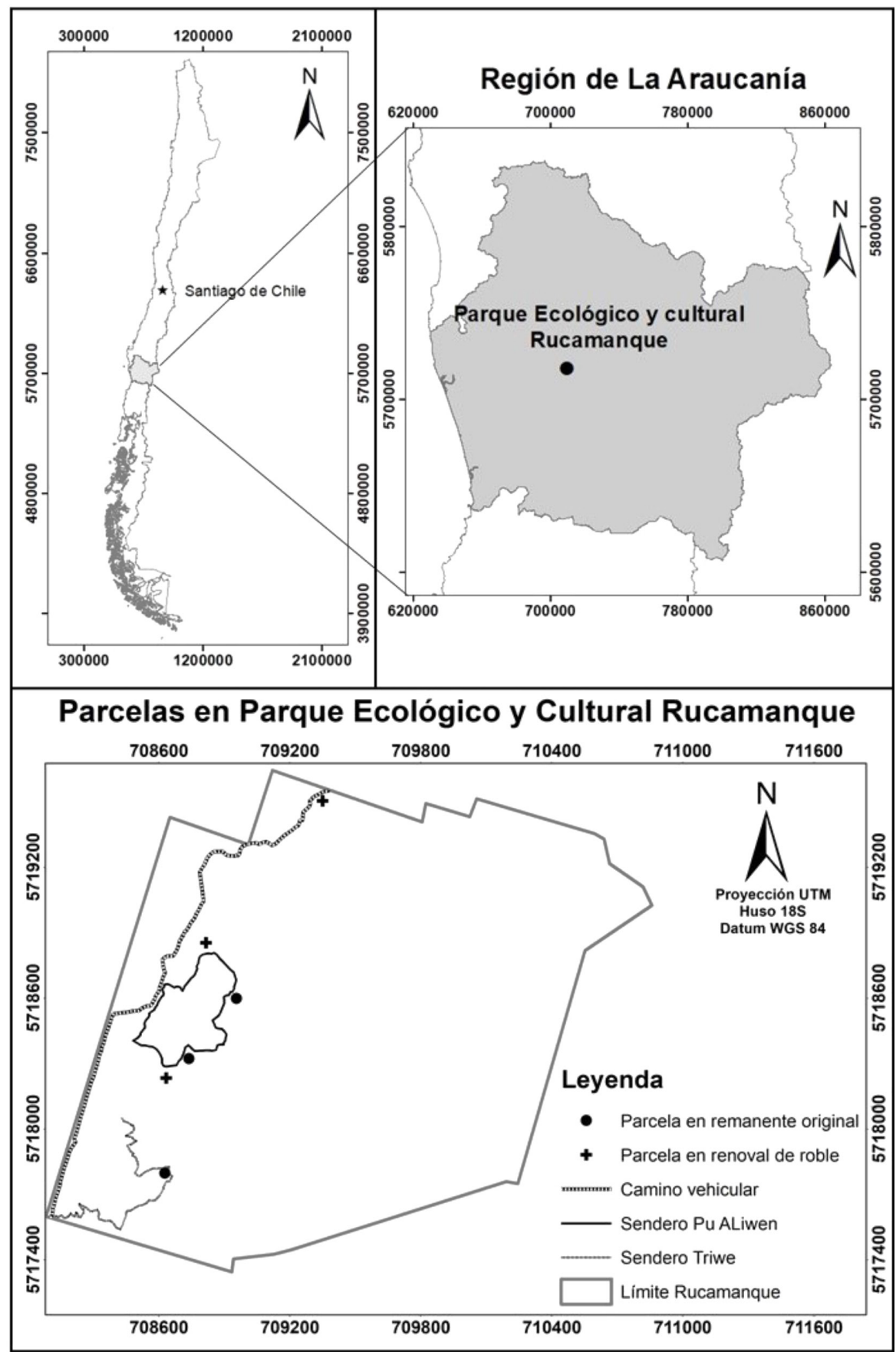

Figura 1. Ubicación geográfica del área de estudio en el Parque Ecológico y Cultural Rucamanque, Región de La Araucanía, Chile. / Geographic location of the study area in the Ecological and Cultural Park Rucamanque in the Araucania Region, Chile. 


\section{RESULTADOS}

Se identificaron 13 especies de líquenes epífitos pertenecientes a cinco familias (Collemataceae, Nephromataceae, Lobariaceae, Pannariaceae y Peltigeraceae) y seis géneros (Leptogium, Pseudocyphellaria, Sticta, Nephroma, Physma y Peltigera; Tabla 1). El mayor número de especies lo presenta la familia Lobariaceae, la que alcanza 9 taxones. El género más representado fue Pseudocyphellaria con seis taxones, seguido de Sticta con tres especies, el resto de los géneros registró solo una especie (Tabla 1). La mayor parte de las especies (46\%) tiene distribución endémica, y un $23 \%$ es cosmopolita (Tabla 1). La mayor riqueza y número de registros fue observada en el Renoval de Roble (estadísticamente significativa, Kruskal-Wallis Test, $H C=4,891 ; P=0,027$ ) con 13 especies a diferencia del Bosque Remanente Original (7 especies en total). En el Renoval de Roble el Índice de Simpson $(0,5604)$ fue menor que en el Bosque Remanente (0,7771); sin embargo, un caso contrario se observó en el índice de Shanon, que fue mayor en el Renoval de Roble
$(1,996)$ que en el Remanente Original $(1,188)$, a diferencia con lo que ocurre con el valor de dominancia que fue mayor en el Bosque Remanente (Tabla 2). El estudio de la presencia de líquenes en distintas alturas del fuste, mostró en general que tanto el número de registros, así como la riqueza no varía estadísticamente $(\mathrm{HC}=9,329$ y $\mathrm{P}=0,096)$ entre las diferentes alturas (Tabla 2), encontrándose el mayor número de registros entre los $75-100 \mathrm{~cm}$. Considerando la riqueza de especies entre los distintos forófitos, se pudo constatar que las diferencias son estadísticamente significativas (KruskalWallis Test, $\mathrm{HC}=7,984$ y $\mathrm{P}=0,046$ ) (Tabla 2), donde Nothofagus obliqua (11 especies) presenta los mayores valores frente a las otras tres especies arbóreas, con 5 en $\mathrm{N}$. dombeyi y Persea lingue y sólo 2 en Aextoxicum punctatum (Tabla 2) siendo los índices de Shanon y Simpson igualmente altos en N. obliqua $(1,953$ y 0,784$)$ que en los otros forófitos. Comparando entre los forófitos, la comunidad de N. obliqua es distinta y estadísticamente significativa (Duncan Test, $\mathrm{P}=0,005841$ ) sólo de A. punctatum.

TABLA 1: Especies de líquenes en el Parque Ecológico y Cultural Rucamanque, Región de La Araucanía tanto en el Renoval de Roble y Remanente Original. $\mathrm{FB}$ = fotobionte ( $\mathrm{C}=$ cianolíquenes / $\mathrm{A}=$ clorolíquenes). / Lichen species in the Rucamanque Ecological and Cultural Park, La Araucanía Region, both in the Renoval de Roble and Remanente Original. FB = photobiont ( $\mathrm{C}=\mathrm{cyanolichens} / \mathrm{A}=\mathrm{chlorolichens}$.

\begin{tabular}{|c|c|c|c|c|c|}
\hline Familia & Especies & FB & $\begin{array}{c}\text { Renoval de } \\
\text { Roble }\end{array}$ & $\begin{array}{c}\text { Remanente } \\
\text { Original }\end{array}$ & $\begin{array}{l}\text { Distribución } \\
\text { geográfica* }\end{array}$ \\
\hline Collemataceae & Leptogium sp. & & $\mathrm{x}$ & $\mathrm{x}$ & Sin información \\
\hline Lobariaceae & Pseudocyphellaria crocata agg. (L.) Vainio & $\mathrm{C}$ & $x$ & $x$ & Cosmopolita \\
\hline Lobariaceae & Pseudocyphellaria flavicans (J. D. Hook \& Taylor) Vainio & A & $x$ & & Endémico \\
\hline Lobariaceae & Pseudocyphellaria hirsuta (Mont.) Malme & $\mathrm{C}$ & $x$ & & Endémico \\
\hline Lobariaceae & Pseudocyphellaria meyenii (Trevisan) D. Galloway & $\mathrm{C}$ & $x$ & $x$ & Endémico \\
\hline Lobariaceae & Pseudocyphellaria nitida (Taylor) Malme & A & $x$ & $x$ & Endémico \\
\hline Lobariaceae & Pseudocyphellaria valdiviana (Nyl.) Follmann & A & $x$ & & Endémico \\
\hline Lobariaceae & Sticta caulescens De Not & $\mathrm{C}$ & $x$ & & Endémico \\
\hline Lobariaceae & Sticta fuliginosa (Dicks.) Ach. & $\mathrm{C}$ & $x$ & $x$ & Cosmopolita \\
\hline Lobariaceae & Sticta weigelii Isert ex Ach. & $\mathrm{C}$ & $x$ & $x$ & Tropical \\
\hline Nephromataceae & Nephroma cellulosum var. cellulosum (Sm.) Ach. & $\mathrm{C}$ & $x$ & & Austral \\
\hline Pannariaceae & Physma chilense Hue & $\mathrm{C}$ & $x$ & $x$ & Austral \\
\hline Peltigeraceae & Peltigera polydactylon (Neck.) Hoffm. & $\mathrm{C}$ & $x$ & & Cosmopolita \\
\hline
\end{tabular}

*Austral (especies con distribución neoaustral y paleoaustral); Cosmopolita (especies con distribución mundial); Endémico (aquellas especies presentes únicamente en el sur de Sudamérica); Tropical (incluye a las especies neotropicales y especies tropicales de distribución más amplia) de acuerdo a Galloway (2008). 


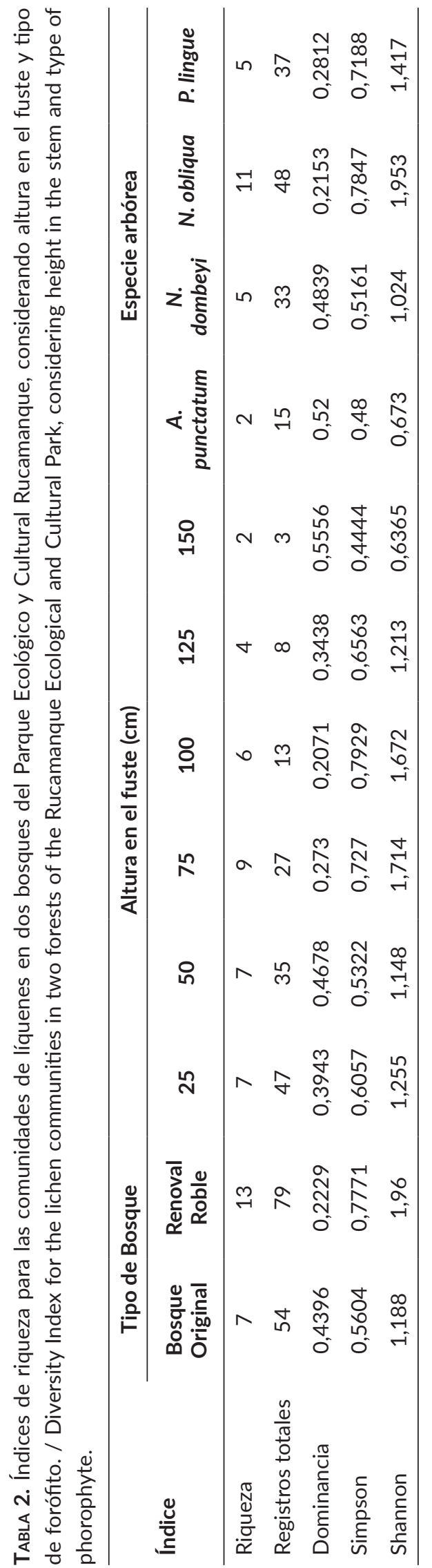

\section{DISCUSIÓN}

Los resultados que aporta este estudio representan una primera aproximación acerca de la diversidad de Peltigerales presentes en un bosque del Tipo Forestal Roble-RaulíCoigüe en la depresión intermedia, una zona donde quedan muy pocos remanentes del bosque original. Las especies de líquenes reportadas para el Parque Rucamanque se encuentran principalmente dentro del elemento valdiviano (Redón 1976) que se caracteriza por la predominancia de especies de la familia Lobariaceae. En el caso de las especies del género Pseudocyphellaria, cabe destacar algunos reportes recientes, que ponen en duda particularmente la presencia del taxón Pseudocyphellaria crocata en Chile (Lücking et al. 2017); estos autores analizaron con técnicas moleculares al menos 50 especímenes de diversos orígenes, demostrándose que $P$. crocata no correspondería a una sola especie, sino que incluye al menos 13 especies distintas, de las cuales 5 de ellas estarían registradas en Chile. Sin embargo, y dado que para los taxones chilenos no se ha realizado una revisión actualizada del género, consideramos que aún no se puede descartar su presencia en Chile como lo indican Vargas \& Sandoval (2020). Una situación similar existe en el caso de la especie Pseudocyphellaria flavicans que ha sido revisada y cambiado su nombre a Podostictina flavicans (Hook. f. \& Taylor) Moncada \& Lücking (Moncada et al. 2014), sin embargo, como en las bases de datos más reciente de los Líquenes de Chile aún permanece con su nombre antiguo (GBIF, Vargas \& Sandoval 2020) hemos adoptado el mismo concepto. En el caso de Pseudocyphellaria meyenii se reporta por primera vez para la Región de la Araucanía (Galloway 1992), ampliándose su distribución. En cuanto al tipo de fotobionte (Tabla 1), encontramos que el $75 \%$ de las especies son cianolíquenes. La alta presencia de cianolíquenes sugiere una gran estabilidad ecológica en este bosque (Gatica et al. 2011) ya que los cianolíquenes tienen una gran sensibilidad ecológica como por ejemplo a prácticas forestales inadecuadas (Richardson \& Cameron 2004; Cameron \& Richardson 2006). Claramente, los nuevos antecedentes reportados aquí sumado a los hallazgos sobre diversas especies de Pseudocyphellaria (Moncada et al. 2013, 2014; Lücking et al. 2017a; Lücking et al. 2017b) implican que se requiere actualizar y revisar las especies presentes en el país.

Por otra parte, encontramos que un $46 \%$ de las especies registradas en este estudio en el Parque Rucamanque de la Comuna de Temuco son endémicas del sur de Sudamérica (Tabla 1) y que están asociadas principalmente al bosque nativo (Galloway 1989, 1992, 1994; White \& James 1988), por lo que es necesario avanzar en describir la biota de estos remanentes de Bosque nativo, para poder estimar su valor 
de conservación. Cabe destacar que los líquenes endémicos, debido a su distribución más restringida, son más vulnerables frente a la pérdida de área climáticamente adecuada aumentando su vulnerabilidad a la extinción como ha sido mostrado en estudios realizados en EEUU y Canadá (Allen \& Lendemer 2016, Allen et al. 2019). La riqueza de especies de líquenes es dependiente de la especie arbórea, con variaciones entre los distintos forófitos, siendo Nothofagus obliqua el que presenta mayor número de especies de líquenes seguida por N. dombeyi y Persea lingue (11 y 5, respectivamente). Aunque en nuestro caso no hemos podido realizar análisis de $\mathrm{pH}$ de la corteza de los forófitos en estudio, se ha reportado previamente que la variación de la riqueza de líquenes por especie hospedera puede estar determinada por esta variable (Mezaka et al. 2012; Li et al. 2015). Sin embargo, aunque Pereira et al. (2014) mostraron que el pH de la corteza de $N$. dombeyi es mucho más ácida que la de N. obliqua, no se pudo establecer una dependencia estrecha entre la riqueza de especies y el $\mathrm{pH}$. Es decir, se requiere analizar otras variables para establecer los patrones que determinan la diversidad de líquenes en estos bosques.

En cuanto al tipo de bosque, encontramos una mayor riqueza de líquenes en el Renoval de Roble (13 especies) frente al Remanente Original en el que se registraron 7 especies (Tabla 1). Como posible explicación a la mayor diversidad en el Renoval de Roble está el dosel abierto que se ha visto que afecta positivamente la diversidad de líquenes (Moning et al. 2009; Pinho et al. 2012) incluso que formaciones boscosas con un manejo que implica perturbación moderada presenta mayor diversidad de líquenes que un bosque original con las mismas especies arbóreas (Pinho et al. 2012). Por otra parte, es muy importante recalcar, que este estudio está focalizado en un solo orden, Peltigerales y que no se han incluido otros taxones. Sin embargo, es probable que este patrón de riqueza pueda cambiar si se trabaja incluyendo en el estudio otros biotipos (crustosos, fruticulosos) y grupos de líquenes.

Las diferencias de diversidad encontradas entre estos dos tipos de bosques pueden explicarse mediante otros factores distintos a la apertura del dosel o el grado de perturbación, como son la edad de los árboles (Armstrong \& Welch 2007; Nascimbene et al. 2009; Johansson et al. 2010), el microclima (Li et al. 2015) o la calidad del área adyacente (Cardós et al. 2016). Sin embargo, también se puede indicar que la altura de los árboles también juega un rol, como ha sido mostrado por Prather et al. (2018), quienes mostraron que un $80 \%$ de la comunidad liquénica puede ser registrada en la parte baja de los árboles (hasta 1,9 m de la base) y un 68\% de la diversidad se encuentra representada en la parte del dosel de árboles de altura mayores a $50 \mathrm{~m}$. Esto podría implicar que para tener una mayor certeza acerca de la diversidad liquénica, se requiere incluir otros parámetros y técnicas para su recolección. Este es un trabajo preliminar y los autores sugieren realizar un estudio más profundo de la flora liquénica asociada a este remanente de la depresión intermedia en el Centro-Sur de Chile, el cual está altamente amenazado (San Martín et al. 1991). Comparar bosques nativos con renovales potencialmente, será de gran ayuda para evaluar a largo plazo posibles impactos generados por cambios tanto globales como locales en los bosques, sobre todo en nuestro país, donde se ha perdido y reemplazado el bosque nativo y no se conocen las comunidades de líquenes originales.

\section{CONCLUSIONES}

Nuestros resultados muestran que hay diferencias en la riqueza respecto de los distintos forófitos y tipos de bosque. Se requiere profundizar en el estudio de estas comunidades de líquenes sobre todo en bosques naturales para tener una línea base para comprender los cambios en estas comunidades frente al escenario de cambio climático.

\section{AGRADECIMIENTOS}

Los autores agradecen al proyecto FONDECYT de Postdoctorado 321056, a la Universidad de la Frontera y administración del Parque Ecológico y Cultural Rucamanque, a Daniela Pérez Paz (guardaparque) y Catalina Marín por la ayuda en el análisis de datos. Se agradece las críticas y comentarios del revisor Sr. Reinaldo Vargas que ayudaron a mejorar este estudio.

\section{REFERENCIAS}

Allen, J.L., Lendemer, J.C. 2016. Climate change impacts on endemic, high-elevation lichens in a biodiversity hotspot. Biodiversity and Conservation 25(3): 555-568.

Allen, J.L.R., McMullin, T., Tripp, E.A., Lendemer, J.C. 2019. Lichen conservation in North America: a review of current practices and research in Canada and the United States. Biodiversity and Conservation 28: 3103-3138.

Armstrong, R.A., Welch, A.R. 2007. Competition in lichen communities. Symbiosis 43: 1-12.

Asplund, J., Wardle, D.A. 2016. How lichens impact on terrestrial community and ecosystem properties. Biological Reviews 1-19.

Cameron, R., Richardson, D. 2006. Occurrence and abundance of epiphytic cyanolichens in protected areas of Nova Scotia, 
Canada. Opuscula Philolichenum 3: 5-14.

Cardós, J.L.H., Martínez, I., Calvo, V., Aragón, G. 2016. Epiphyte communities in Mediterranean fragmented forests: Importance of the fragment size and the surrounding matrix. Landscape Ecology 31: 1975-1995.

Colwell, R.K., Mao, C.X., Chang, J. 2004. Interpolating, extrapolating, and comparing incidence-based species accumulation curves. Ecology 85: 2717-2727.

Di Castri, F., Hajek, E. 1976. Bioclimatología de Chile. Universidad Católica de Chile. Santiago. 128 pp.

Donoso, C. 1981. Tipos Forestales de los Bosques Nativos de Chile. Investigación y Desarrollo Forestal (CONAF/ PNUD/FAO) Documento de Trabajo Nº 38 (Publicación Fao Chile). Santiago. Chile. 82 pp.

Donoso, C. 1983. Modificaciones del paisaje forestal chileno a lo largo de la historia. En: Simposium Desarrollo y Perspectivas de las Disciplinas Forestales de la Universidad Austral de Chile. 365-438 pp.

Fanning, E.F., Ely, J.S., Lumbsch, H.T., Keller, H.W. 2007. Vertical distribution of lichen growth forms in tree canopies of Great Smoky Mountains National Park. Southeast. Nat. Special Issue 1: 83-88.

Fernández, I., Morales, N., Olivares, L., Salvatierra, J., Gómez, M., Montenegro, G. 2010. Restauración ecológica para ecosistemas nativos afectados por incendios forestales. Facultad de Agronomía e Ingeniería Forestal. Pontificia Universidad Católica de Chile, Santiago, Chile.

Galloway, D.J. 1989. Studies in Pseudocyphellaria (lichens) I. The New Zealand species. Bulletin of the British Museum (Natural History), Botany 17(1): 1-267.

Galloway, D.J. 1992. Studies in Pseudocyphellaria (lichens) III. The South American species. Bibliotheca Lichenologica. J. Cramer, Berlín. 275 pp.

Galloway, D.J. 1994. Studies on the lichen genus Sticta (Schreber) Ach.: I. Southern South American species. The Lichenologist 26(3): 223-282.

Galloway, D.J. 2008. Lichen biogeography. En: Nash, T.H. (Ed.). Lichen Biology: 315-335. Second Edition. Cambridge University Press, Cambridge.

Gatica, A., Pereira, I., Vallejos, O. 2011. Epiphytic lichens: A tool for to study the ecological continuity Mocha Island, Chile. Gayana Botánica 68: 226-235.

Hajek, E., Di Castri, F. 1975. Bioclimatografía de Chile. Universidad Católica de Chile, Santiago. 214 pp.

Johansson, V., Snäll, T., Johansson, P., Ranius, T. 2010. Detection probability and abundance estimation of epiphytic lichens based on height-limited surveys. Journal of Vegetation Science 21: 332-341.

Lesica, P., McCune, B., Cooper, S.V., Hong, W.S. 1991. Differences in lichen and bryophyte communities between old- growth and managed second growth forests in Swan Valley, Montana. Canadian Journal of Botany 69: 17451755.

Li, S., Liu, W.Y., Li, D.W., Song, L., Shi, X.M., Lu, H.Z. 2015. Species richness and vertical stratification of epiphytic lichens in subtropical primary and secondary forests in southwest China. Fungal Ecology 17: 30-40.

Lücking, R., Hodkinson, B.P., Leavitt, St.D. 2017a. The 2016 classification of lichenized fungi in the Ascomycota and Basidiomycota - Approaching one thousand genera. The Bryologist 119(4): 361-416.

Lücking, R., Moncada, B., McCune, B., Farkas, E., Goffinet, B., Parker, D., Chaves, J.L., Lőkös, L., Nelson, P.R., Spribille, T., Stenroos, S., Wheeler, T., Yanez-Ayabaca, A., Dillman, K., Gockman, O.T., Goward, T., Hollinger, J., Tripp, E.A., Villella, J., Álvaro-Alba, W.R., Arango, C.J., Cáceres, M.E.S, Coca, L.F., Printzen, C., Rodríguez, C., Scharnagl, K., Rozzi, R., Soto-Medina, E.D., Yakovchenko, L.S. 2017b. Pseudocyphellaria crocata (Ascomycota: Lobariaceae) in the Americas is revealed to be thirteen species, and none of them is P. crocata. The Bryologist 120(4): 441-500.

Mezaka, A., Brumelis, G., Piterans, A. 2012. Tree and stand-scale factors affecting richness and composition of epiphytic bryophytes and lichens in deciduous woodland key habitats. Biodiversity and Conservation 21: 3221-3241.

Miadlikowska, J., Lutzoni, F. 2004. Phylogenetic classification of peltigeralean fungi (Peltigerales, Ascomycota) based on ribosomal RNA small and large subunits. American Journal of Botany 91: 449-464.

Moncada, B., Reidy, B., Lücking, R. 2014. A phylogenetic revision of Hawaiian Pseudocyphellaria sensu lato (lichenized Ascomycota: Lobariaceae) reveals eight new species and a high degree of inferred endemism. The Bryologist 117(2): 119-160.

Moning, C., Werth, S., Dziock, F., Bässler, C., Bradtka, J., Hothorn, T., Müller, J. 2009. Lichen diversity in temperate montane forests is influenced by forest structure more than climate. Forest Ecology and Management 258: 745-75.

Nascimbene, J., Marini, L., Motta, R., Nimis, P.L. 2009. Influence of tree age, tree size and crown structure on lichen communities in mature Alpine spruce forests. Biodiversity and Conservation 18: 1509-1522.

Nash, T.H.III. 2008. Introduction. En: Lichen biology: 1-8. Second edition. Cambridge University Press, Cambridge.

Nuñez, P., Espinosa, A., Pacheco, P. 2015. Parque Ecológico y Cultural Rucamanque. Plan de Manejo Integral. Universidad de La Frontera, Facultad de Ciencias Agropecuarias y Forestales, Departamento de Ciencias Forestales. Temuco, Chile.

Orange, A., James, P.W., White, F.J. 2001. Microchemical 
methods for the identification of lichens. British Lichen Society, London. $101 \mathrm{pp}$.

Pereira, I. 2007. Micobiota líquenizada del Parque Katalapi, $X$ Región, Chile. Gayana Botánica 64(2): 192-200.

Pereira, I., Mueller, F., Moya, M. 2014. Influence of Nothofagus bark $\mathrm{pH}$ on the lichen and bryophytes richness, Central Chile. Gayana Botánica 71(1): 120-130.

Pereira, I., Wang, X., Oh, S., Sánchez, P., Hur, J. 2016. Líquenes de los alrededores de las Termas de Chillán y Las Trancas, Región del Bío-Bío, Chile. Gayana Botánica 73(1): 104112.

Pinho, P., Bergamini, A., Carvalho, P., Branquinho, C., Stofer, S., Scheidegger, C., Máguas, C. 2012. Lichen functional groups as ecological indicators of the effects of land-use in Mediterranean ecosystems. Ecological Indicators 15: 36-42.

Prather, H.M., Eppley, S.M., Rosenstiel, T.N. 2018. Urban forested parks and tall tree canopies contribute to macrolichen epiphyte biodiversity in urban landscapes. Urban Forestry and Urban Greening 32: 133-142.

Quilhot, W., Cuellar, M., Díaz, R., Riquelme, F., Rubio, C. 2010. Estudio preliminar de la flora liquénica de Isla Mocha, sur de Chile. Gayana Botánica 67(2): 206-212.

Quilhot, W., Cuellar, M., Díaz, R., Riquelme, F., Rubio, C. 2012. Lichens of Aysen, Southern Chile. Gayana Botánica 69(1): 57-87.

Redón, J. 1976. Fitogeografía de los líquenes chilenos. Anales del Museo de Historia Natural de Valparaíso 9: 7-22.
Redón, J., Arellano, L., Riveros, M. 1979. Los líquenes de Cordillera Pelada. I: Estudio preliminar. Medio Ambiente 4: 71-79.

Richardson, D.H.S., Cameron, R.P. 2004. Cyanolichens: their response to pollution and possible management strategies for their conservation in northeastern. Naturalist 11: 1-22.

Rubio, C., Saavedra, M., Cuellar, M., Díaz, R., Quilhot, W. 2013. Epiphytic lichens of Conguillío National Park, Southern Chile. Gayana Botánica 70(1): 66-81.

Salas, C. 2001. Caracterización básica del relicto de biodiversidad Rucamanque. Bosque Nativo 29: 3-9.

San Martín, C., Ramírez, C., Figueroa, H., Ojeda, N. 1991. Estudio sinecológico del bosque de Roble-Laurel-Lingue del Centro-Sur de Chile. Bosque 12: 11-27.

Sporn, S.G., Bos, M.M., Kessler, M. 2010. Vertical distribution of epiphytic bryophytes in an Indonesian rainforest. Biodiversity and Conservation 19: 745-760.

Van Stan, J.T., Pypker, T.G. 2015. A review and evaluation of forest canopy epiphyte roles in the partitioning and chemical alteration of precipitation. Science of the Total Environment 536: 813-824.

Vargas Castillo, R., Sandoval Leiva, P. 2020. Lista sistemática de los hongos liquenizados y liquenícolas presentes en Chile. Version 1.6. Universidad Metropolitana de Ciencias de la Educación. Checklist dataset https://doi.org/10.15468/ jxynx2 Accedido: Septiembre 17, 2020 via GBIF.org

White, F.J., James, P.W. 1988. Studies on the Genus Nephroma II. The Southern Temperate Species. The Lichenologist 20: 103-166.

Received: 05.05.2020

Accepted: 10.03.2021 